June 24th, 2016

\begin{tabular}{|c|c|c|}
\hline Title: & \multicolumn{2}{|c|}{$\begin{array}{l}\text { Improving dynamics of repetitive control based current harmonic mitigation in inverter-fed } \\
\text { permanent magnet synchronous machines with nonlinear magnetics }\end{array}$} \\
\hline Authors: & \multicolumn{2}{|c|}{ Jan Richter, Martin Doppelbauer } \\
\hline Institute: & \multicolumn{2}{|c|}{$\begin{array}{l}\text { Karlsruhe Institute of Technology (KIT) } \\
\text { Elektrotechnisches Institut (ETI) - Hybrid Electric Vehicles (HEV) }\end{array}$} \\
\hline Type: & \multicolumn{2}{|c|}{ Conference Proceedings } \\
\hline Published at: & $\begin{array}{l}\text { The } 8^{\text {th }} \text { IET Internation } \\
\text { (PEMD) } 2016 \\
\text { Date of Conference: } \\
\text { Conference Location: } \\
\text { Publisher: } \\
\text { ISBN: } \\
\text { DOI: }\end{array}$ & $\begin{array}{l}\text { Conference on Power Electronics, Machines and Drives } \\
\text { 19. April } 2016-21 . \text { April } 2016 \\
\text { Glasgow, Scotland, UK } \\
\text { The Institution of Engineering and Technology (IET) } \\
978-1-78561-189-6 \\
\underline{10.1049 / c p .2016 .0166}\end{array}$ \\
\hline
\end{tabular}




\title{
Improving dynamics of repetitive control based current harmonic mitigation in inverter-fed permanent magnet synchronous machines with nonlinear magnetics
}

\author{
Jan Richter*, Martin Doppelbauer \\ Institute of Electrical Engineering (ETI), Karlsruhe Institute of Technology (KIT) \\ Kaiserstr. 12, 76131 Karlsruhe, Germany, *jan.richter@kit.edu
}

Keywords: Harmonics, Machine Control, Permanent Magnet Motors, Power Electronics, Variable Speed Drives.

\begin{abstract}
Inverter nonlinearities and machine spatial harmonics yield unwanted machine current harmonics causing noise, vibration, additional losses and torque ripples. In this paper the dynamics of a repetitive control based mitigation method are analysed. The proposed scheme identifies voltage errors and compensates their effects online for interior permanent magnet synchronous machines with nonlinear magnetics. It requires no additional sensors and only fundamental machine model parameters. Test bench measurements of maximal torque inversion and variable speed operation proof the fast convergence of the developed algorithm. Current harmonic mitigation is reached after several electric periods and in the time range of $100 \mathrm{~ms}$ during typical operational conditions. The functional principle is transferable to other machine types or grid applications enabling fast and precise mitigation of current harmonics in a wide field of applications.
\end{abstract}

\section{Introduction}

The influences of machine current harmonics in inverter-fed drives are well known. They generate additional losses, torque ripples, noise, vibration and control loop instabilities. Current harmonics are caused by three effects: inverter switching, inverter nonlinearities and spatial harmonics of the machine. Current harmonics due to inverter switching are unavoidable because they directly follow the used working principle of the $\mathrm{DC} / \mathrm{AC}$ inverter and the pulse-frequency related modulation scheme. However, current harmonics caused by inverter nonlinearities and spatial harmonics of the machine can be mitigated.

A whole class of mitigation methods arises from the fact that current harmonics have a repetitive nature during stationary operation. Thus, a straight forward approach is to identify the effects in one electric period and feed-forward them in the next. This simple principle originates from self-learning algorithms [1] and is called repetitive control. It is used in numerous applications [2] and also attracts attention in power electronics. In grid-connected inverters voltage [3] and current harmonics [4] were mitigated using repetitive controllers. Application of the principle to inverter-fed variable speed drives is also possible and enables mitigation $[5,6]$ and control of machine current harmonics [7]. Indeed, an intrinsic problem of repetitive control based controllers is the slow system response because on the one hand controller gains have to be chosen carefully to ensure stability and on the other hand a delay of one electric period is introduced between identification and feed-forward control. This major drawback hinders wide

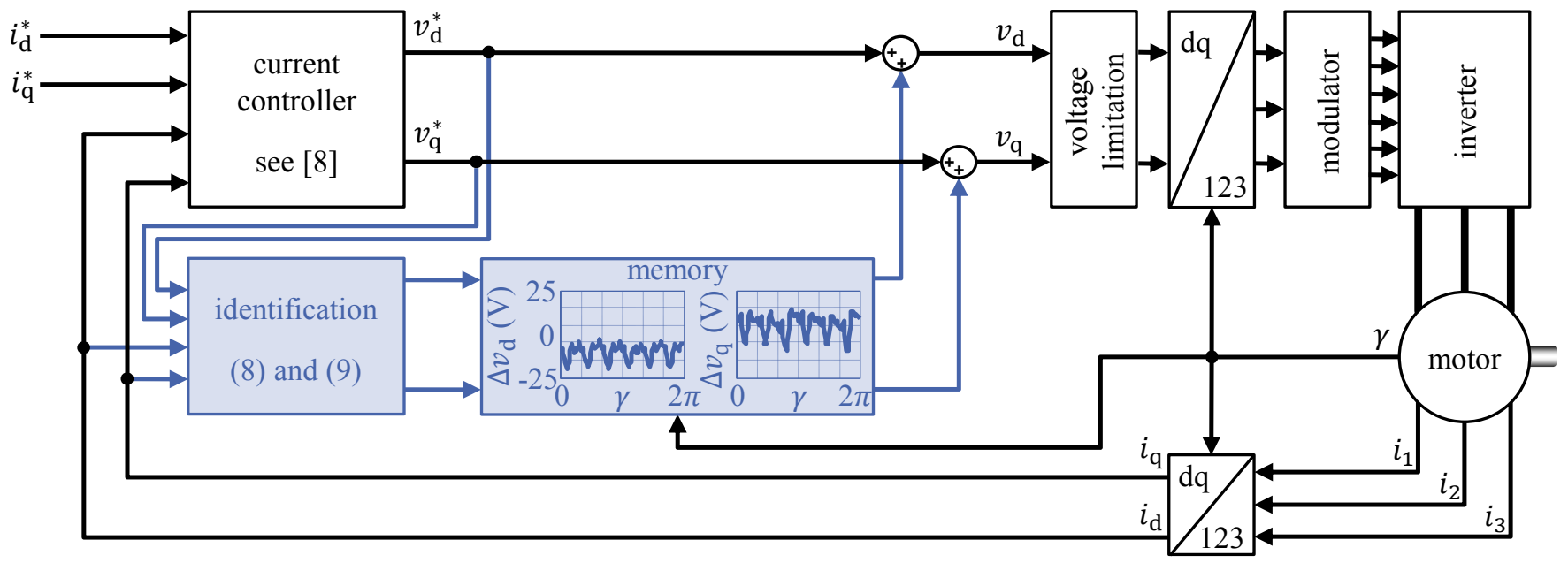

Fig. 1: Scheme of the presented method. A conventional current control structure is used for the currents $i_{x}$ that are measured and closed loop controlled to the reference values $i_{x}^{*}$ with $x \in\{\mathrm{d}, \mathrm{q}\}$. The presented method is shadowed blue. Voltage errors $\Delta v_{x}$ are identified online, stored in dependence on the rotor angle $\gamma$ and fed-forward. 
application of repetitive controllers in inverter-fed drives and is addressed in the following.

As will be demonstrated, current harmonics are caused by voltage errors of the inverter and the machine. Two features are required to consider voltage errors in the controller designs as illustrated by the two blue blocks in Fig. 1:

- The voltage errors $\Delta v_{x}$ have to be identified both fast and precisely by using measured or known quantities.

- They are stored in a memory in dependence on the rotor angle $\gamma$ and fed-forward. When the current reference values $i_{x}^{*}$ change, the memory has to be initialized wisely.

The proposed method is integrated in a standard rotor-oriented current control structure. A dead-beat current controller that does not contain an integral part but uses the reference and measured currents to calculate the controller output voltage $v_{x}^{*}$ is applied [8]. After back-transformation to the stator-fixed reference frame, a modulator creates the gate signals for the two-level DC/AC inverter that operates at constant switching frequency. The controller can mitigate all current harmonics that are integer multiples of the fundamental frequency. Mitigation performance is only restricted by the physical limits of the drive system given by the switching frequency and the voltage limit of the inverter. The number of required calculations and the need of additional dynamic memory is very low and allows an easy implementation on microcontrollers. The dynamic performance of the proposed control method is demonstrated by means of an automotive traction interior permanent magnet synchronous machine with nonlinear magnetics.

In the next section the machine and inverter models are covered. Then the online identification and harmonic mitigation scheme is developed theoretically. Details on implementation and the used test bench are described in Section 3. Dynamic measurements are presented and discussed in Section 4 and concluded in Section 5.

\section{Theory}

The basic theory of online voltage error identification in inverted-fed machines with nonlinear magnetics was already described in [6] and enhanced in [9]. In the following it is briefly summarized with the focus set on dynamic behaviour and methods to obtain a faster system response.

\subsection{Machine and Inverter Model}

Validated models of permanent magnet synchronous machines that include the effects of magnetic rotor anisotropy and nonlinear magnetics due to iron saturation and spatial harmonics are described in literature [10] and applied in the following. It is assumed that the machine features three symmetric star-connected phases with the neutral point not connected to the inverter. Dielectric currents, temperature dependencies as well as skin, proximity and aging effects are disregarded. Moreover, iron and friction losses are not considered. The neglected effects could be taken into account yielding more complicated models and additional parameter variations. As shown in Section 4, this is not necessary because the total model error introduced by all approximations is sufficiently small to enable fast and precise mitigation of current harmonics.

By employing Ohm's law, Faraday's law of induction and Kirchhoff's laws to the machine's coils, the stator voltages can be calculated. Subsequent transformation to the rotor-fixed dqreference frame yields:

$$
\begin{aligned}
& v_{\mathrm{d}}=R i_{\mathrm{d}}+\frac{\mathrm{d} \Psi_{\mathrm{d}}}{\mathrm{d} t}-\omega \Psi_{\mathrm{q}} \\
& v_{\mathrm{q}}=R i_{\mathrm{q}}+\frac{\mathrm{d} \Psi_{\mathrm{q}}}{\mathrm{d} t}+\omega \Psi_{\mathrm{d}}
\end{aligned}
$$

Therein $t$ denotes the time, $R$ the ohmic stator resistance, $\omega$ the electric angular frequency, $v_{x}$ the stator voltage, $i_{x}$ the stator current and $\Psi_{x}$ the stator flux linkage with $x \in\{\mathrm{d}, \mathrm{q}\}$. The stator resistance and the flux linkages are required as model parameters. The stator resistance can be determined by a DC measurement at motor standstill. Though, the flux linkages $\Psi_{x}$ depend nonlinearly on the currents because of magnetic anisotropy, iron and cross-saturation and also on the rotor angle $\gamma$ due to spatial harmonics. When spatial harmonics are interpreted and considered as voltage errors as shown below, the flux linkages can be averaged over the rotor angle $\gamma$. The flux linkages then become two-dimensional as defined by the function $f$.

$$
f: \mathbb{R}^{2} \rightarrow \mathbb{R}^{2},\left(i_{\mathrm{d}}, i_{\mathrm{q}}\right) \mapsto\left(\Psi_{\mathrm{d}}, \Psi_{\mathrm{q}}\right)
$$

The function $f$ can be obtained from either finite element analysis or measurements at stationary operation. When the currents are constant, the stationary machine equations can be solved for the flux linkages. All other quantities are measured [11]. Repeating the latter at different currents results in $f$ as depicted for the machine under test in Fig. 2 (a) and (b). The flux linkage $\Psi_{\mathrm{d}}$ changes differently with $i_{\mathrm{d}}$ than $\Psi_{\mathrm{q}}$ does with $i_{\mathrm{q}}$ because of the magnetic anisotropy of the rotor. Both relations are nonlinear due to iron saturation. In addition, crosssaturation leads to a variation of $\Psi_{\mathrm{d}}$ with $i_{\mathrm{q}}$ and of $\Psi_{\mathrm{q}}$ with $i_{\mathrm{d}}$. In order to enable application of the motor model in digital (a)

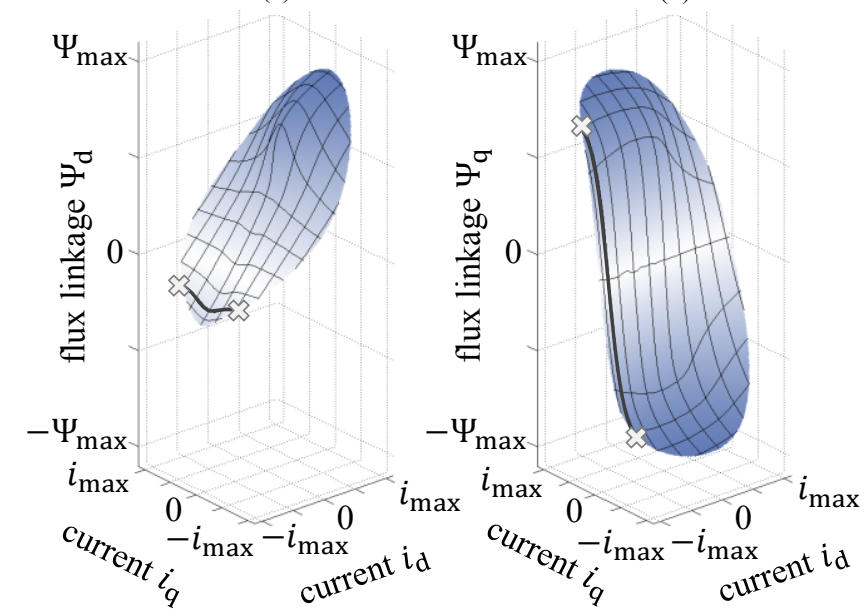

Fig. 2: Nonlinear flux linkage function $f$ for the machine under test. The flux linkages $\Psi_{\mathrm{d}}$ are displayed in (a) and $\Psi_{\mathrm{q}}$ in (b) with $i_{\max }=400 \mathrm{~A}$ and $\Psi_{\max }=0.2 \mathrm{Vs}$. The influences of nonlinear magnetics are clearly visible. The measured torque step from minimal to maximal torque of Fig. 4 is marked grey. 
controllers (1) and (2) have to be discretized in time. Therefore the trapezoidal rule is used in between the points in time $t_{n-1}$ and $t_{n}$ with $n \in \mathbb{N}$ [9]. All angular effects due to spatial harmonics are defined as voltage errors $\Delta v_{x \text {,machine, } n-1}$. Then, the flux linkages $\Psi_{x, n-1}$ and $\Psi_{x, n}$ can be calculated by (3) inserting the measured currents $i_{x, n-1}$ and $i_{x, n}$.

$$
\begin{aligned}
v_{\mathrm{d}, n-1}= & \frac{1}{2} R\left(i_{\mathrm{d}, n-1}+i_{\mathrm{d}, n}\right)+\frac{\Psi_{\mathrm{d}, n}-\Psi_{\mathrm{d}, n-1}}{T} \\
& -\frac{1}{2} \omega\left(\Psi_{\mathrm{q}, n-1}+\Psi_{\mathrm{q}, n}\right)+\Delta v_{\mathrm{d}, \text { machine }, n-1} \\
v_{\mathrm{q}, n-1}= & \frac{1}{2} R\left(i_{\mathrm{q}, n-1}+i_{\mathrm{q}, n}\right)+\frac{\Psi_{\mathrm{q}, n}-\Psi_{\mathrm{q}, n-1}}{T} \\
& +\frac{1}{2} \omega\left(\Psi_{\mathrm{d}, n-1}+\Psi_{\mathrm{d}, n}\right)+\Delta v_{\mathrm{q}, \text { machine }, n-1}
\end{aligned}
$$

Therein $T=t_{n+1}-t_{n}$ denotes the control period duration. Equations (4) and (5) completely describe the dynamic machine behaviour considering rotor anisotropy, iron saturation and spatial harmonics. As model parameters only the stator resistance and fundamental flux linkages, as defined by (3), are required because angular dependencies are interpreted as voltage errors $\Delta v_{x, \text { machine, } n-1}$.

The stator voltages $v_{x, n-1}$ of the machine are supplied by an inverter. Though, the inverter is a nonlinear voltage source because the inverter output voltages $v_{x, n-1}$ differ from the controller reference voltages $v_{x, n-1}^{*}$. The differences are defined as inverter voltage errors $\Delta v_{x, \text { inverter, } n-1}$. In order to consider voltage errors, a simple time-dependent voltage source in the rotor-oriented dq-reference frame is used as inverter model.

$$
\begin{aligned}
& v_{\mathrm{d}, n-1}=v_{\mathrm{d}, n-1}^{*}-\Delta v_{\mathrm{d}, \text { inverter }, n-1} \\
& v_{\mathrm{q}, n-1}=v_{\mathrm{q}, n-1}^{*}-\Delta v_{\mathrm{q}, \text { inverter }, n-1}
\end{aligned}
$$

Inverter voltage errors are caused by various effects [12]: forward voltage and differential resistance of diodes and switches, switching time and switching behaviour, dead-times, minimal on- and off-times and current zero-clamping.

\subsection{Identification of Voltage Errors}

The voltage errors $\Delta v_{x, \text { machine, } n-1}$ and $\Delta v_{x, \text { inverter, } n-1}$ are unknown in (4) to (7). Their identification is challenging because the voltage errors vary with the machine and inverter state variables and parameters. Voltage errors depend on the currents $i_{\mathrm{d}}$ and $i_{\mathrm{q}}$, the rotor angle $\gamma$, the rotor speed, the DC link voltage and the diode and switch parameters that change both with temperature and age. Thus, in practical applications a procedure is needed to determine the voltage errors in each control period during drive operation online. Then the dependencies on state variables and parameters are not a problem because voltage error changes are continuously identified and tracked. Since the stator voltages of the machine are supplied by the inverter (4) and (6) as well as (5) and (7) can be equalized and solved for the voltage errors.

$$
\begin{aligned}
\Delta v_{\mathrm{d}, n-1}= & \Delta v_{\mathrm{d}, \text { machine }, n-1}+\Delta v_{\mathrm{d}, \text { inverter }, n-1} \\
= & v_{\mathrm{d}, n-1}^{*}-\frac{1}{2} R\left(i_{\mathrm{d}, n-1}+i_{\mathrm{d}, n}\right)-\frac{\Psi_{\mathrm{d}, n}-\Psi_{\mathrm{d}, n-1}}{T} \\
& +\frac{1}{2} \omega\left(\Psi_{\mathrm{q}, n-1}+\Psi_{\mathrm{q}, n}\right)
\end{aligned}
$$

$$
\begin{aligned}
\Delta v_{\mathrm{q}, n-1}= & \Delta v_{\mathrm{q}, \text { machine }, n-1}+\Delta v_{\mathrm{q}, \text { inverter }, n-1} \\
= & v_{\mathrm{q}, n-1}^{*}-\frac{1}{2} R\left(i_{\mathrm{q}, n-1}+i_{\mathrm{q}, n}\right)-\frac{\Psi_{\mathrm{q}, n}-\Psi_{\mathrm{q}, n-1}}{T} \\
& -\frac{1}{2} \omega\left(\Psi_{\mathrm{d}, n-1}+\Psi_{\mathrm{d}, n}\right)
\end{aligned}
$$

By (8) and (9) the total voltage errors $\Delta v_{x, n-1}$ can be calculated in each control period because all quantities on the right-hand side are known. The currents $i_{x, n-1}$ of the previous and the actual control period $i_{x, n}$ are measured, the flux linkages $\Psi_{x, n-1}$ and $\Psi_{x, n}$ are obtained by (3) and the controller reference voltages $v_{x, n-1}^{*}$ are calculated by the controller. Model parameter errors of the stator resistance and the flux linkages are identified as voltage errors. Since the control scheme compensates voltage errors, model parameter errors are thus corrected as well.

\subsection{Mitigation of Current Harmonics}

If the two blue blocks in Fig. 1 are disabled, voltage errors will be neglected in the controller design. Then, the machine currents will oscillate during stationary operation because both the voltage errors and the currents change from one control period to another. The current controller reacts to the current control deviation resulting in oscillatory behaviour $[6,9]$. To avoid that problem, the voltage errors are to be considered in the controller design which is described next.

The proposed harmonic mitigation method is added in parallel to the current controller and consists of an identification and memory block. As described in Section 2.2 the voltage errors $\Delta v_{x, n-1}$ are identified in each control period by (8) and (9). Direct feed-forward control of the so identified voltage errors does not work because the voltage errors significantly change within the duration of two control periods. Though, during stationary operation the voltage errors only change rapidly with the rotor angle $\gamma$. This is true because all other effects that influence voltage errors do not change, vary with a slow rate or are periodical with the rotor angle. That is why the voltage errors can be stored and fed-forward in dependence on the rotor angle $\gamma$. As shown in Fig. 3, the angles $\gamma_{\text {store }}$ and $\gamma_{\text {read }}$ of the middle of the previous and next control period are used to store and read the voltage errors.

$$
\begin{aligned}
& \gamma_{\text {store }}=\gamma_{n}-\frac{1}{2} \omega T \\
& \gamma_{\text {read }}=\gamma_{n}+\frac{3}{2} \omega T
\end{aligned}
$$

The voltage errors from the memory are added to the controller output voltage and cropped to the inverter hexagon voltage limit. A classic back-calculation path is used as anti-windup for the memory but not included in Fig. 1 for the sake of readability. Inferior dynamic performance of the controller is obvious. An identified voltage error is not fed-forward until the rotor returns to the same angle again. Dynamics can be significantly improved by two enhancements.

First, the voltage errors have to be calculated precisely by (8) and (9). Thereby a very good estimatation of the voltage errors is obtained within the duration of a single control period. The so identified voltage errors are stored in the memory using a damping factor $0<k_{\text {damp }} \leq 1$. 


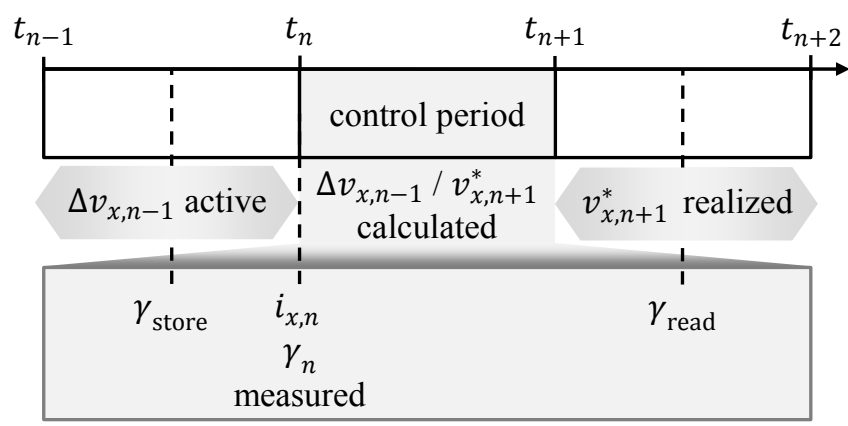

Fig. 3: Timing of measurements and calculations.

$$
\Delta v_{x, \text { new }}\left(\gamma_{\text {store }}\right)=\Delta v_{x}\left(\gamma_{\text {store }}\right)+k_{\text {damp }} \cdot \Delta v_{x, n-1}
$$

Lower values of $k_{\text {damp }}$ yield a slower dynamic response but better noise cancelling. Therefore, the value of $k_{\text {damp }}$ can be adjusted dynamically to improve system behaviour.

Second, the memory is initialised after changes of the machine reference values. The fact that voltage errors dominantly contain harmonics of the order $6 k$ with $k \in \mathbb{N}$ of the fundamental frequency in the rotor-orientated dq-reference frame in applications is applied. For instance, the $-5^{\text {th }}$ and $7^{\text {th }}$ harmonics in the stator-fixed reference frame are often dominant and transformed to a $6^{\text {th }}$ in the dq-reference frame. Thus, after dynamic operation the identified voltage errors of the first sixth of the electric period can be stored at not one but six angles in the memory.

$$
\gamma_{\text {store }, m}=\gamma_{\text {store }}+\frac{2 \pi m}{6} \text { with } m \in\{\mathbb{N} \mid 1 \leq m \leq 6\}
$$

At higher rotor speeds, some rotor angles of the memory might be skipped from one control period to another. But linear interpolation of the identified voltage errors can be used to thoroughly initialize missing rotor angles. Thereby the memory is well initialized after one sixth of the electric period. When the machine reference values change and during the first sixth of the electric period after dynamic operation, the memory is bypassed and the identified voltage errors are directly fed-forward. This improves the dynamic response but also results in feed-forward errors that prove to be tolerable during dynamic operation. The bypass is not depicted in Fig. 1 for the sake of readability. In summary, only the fundamental machine model used for precise voltage error identification combined with improved initialization of the memory yields a fast dynamic response of the repetitive controller in the whole operational area of the machine.

\section{Experimental Setup}

Experiments are conducted with an automotive traction interior permanent magnet synchronous machine of type Brusa HSM16.1712-CO1. The machine properties are given in Table 1 and the nonlinear flux linkage functions in Fig. 2. A speedcontrolled induction machine by Wittur is used as load. Inverters for grid connection and control of both machines are based on Semikron SKiiP 513GD122-3DUL modules. Currents are measured by the built-in Semikron SKiiP current transducers. Speed and rotor angle are calculated using the machine incremental encoder signals.

\begin{tabular}{|l|c|}
\hline \multicolumn{1}{|c|}{ Quantity } & Value \\
\hline voltage nom. & $212 \mathrm{~V}$ \\
\hline current nom. / max. & $169 \mathrm{~A} / 300 \mathrm{~A}$ \\
\hline shaft power nom. / max. & $57 \mathrm{~kW} / 97 \mathrm{~kW}$ \\
\hline speed nom. / max. & $4200 \mathrm{~min}^{-1} / 11000 \mathrm{~min}^{-1}$ \\
\hline torque nom. / max. & $130 \mathrm{Nm} / 220 \mathrm{Nm}$ \\
\hline number of pole pairs & 3 \\
\hline rotor inertia & $0.06 \mathrm{kgm}^{2}$ \\
\hline
\end{tabular}

Table 1: Properties of the motor under test.

The proposed current mitigation method is implemented on the digital signal processor (DSP) TMS320C6748 by Texas Instruments. The function $f$ is stored as lookup table in an external SD-RAM. The voltage errors are stored in the internal DSP memory using 120 rotor angle supporting points resulting in an additional memory demand of 960 bytes at single precision. Supplementary calculation time is almost negligible because only a few additions, subtractions and multiplications of (8) and (9) are performed. To realize a constant switching frequency of $8 \mathrm{kHz}$, all calculations are executed within less than $125 \mu \mathrm{s}$. Inverter gate signals are created by a modulator using space vector modulation and a field programmable gate array (FPGA) of the Cyclone IV series by Altera.

\section{Results and Discussion}

Measurements of current harmonic mitigation of the proposed control scheme during stationary operation in the whole operational area were already published $[6,9]$.

In the following the dynamic behaviour of the described controller is further analysed by means of two experiments: First a maximal torque inversion at half the nominal speed and second an acceleration from standstill to $10.000 \mathrm{~min}^{-1}$ with constant reference currents. In both experiments two different controller setups are compared:

- The controller of Fig. 1 but with disabled identification and memory block. Instead a standard scheme to compensate the inverter dead times is applied [13] (upper / green plots in Fig. 4 and Fig. 5).

- The proposed method of Fig. 1 (lower / blue plots in Fig. 4 and Fig. 5).

\subsection{Maximal Torque Inversion}

The flux linkages of the maximal torque inversion from $-220 \mathrm{Nm}$ to $220 \mathrm{Nm}$ are marked as white crosses and grey lines in Fig. 2 (a) and (b). Magnetics are strongly nonlinear during the torque inversion. According measurement results at half the nominal speed of $2100 \mathrm{~min}^{-1}$ are depicted in Fig. 4. In order to realize the maximal torque inversion the reference direct current stays constant and the quadrature reference current is inverted. As visible in Fig. 4 (a) both controllers execute the torque step of $440 \mathrm{Nm}$ within $2 \mathrm{~ms}$ by using the hexagon voltage limit of the inverter for 16 control periods. It should be noted that the torque change is realized using the physical limit of the inverter. That is why no other controller can reach the 
(a)

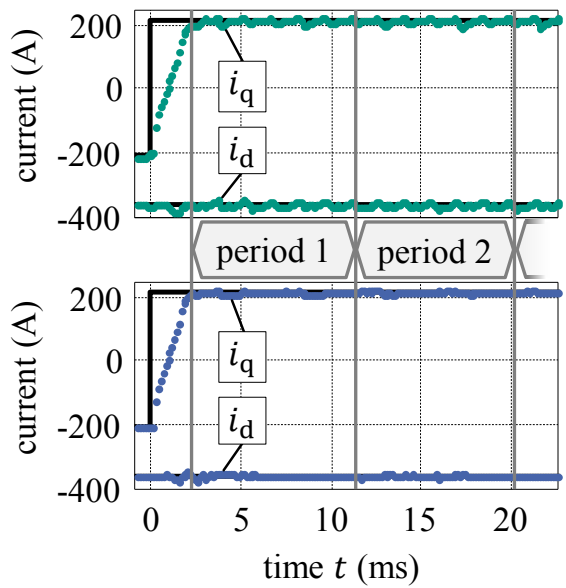

(b)

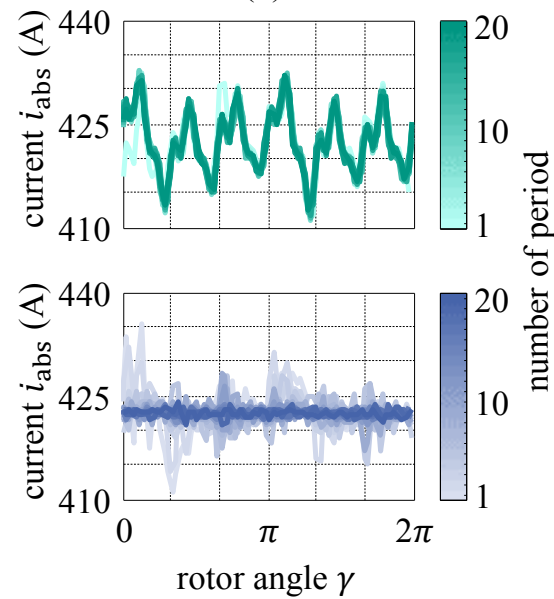

(c)

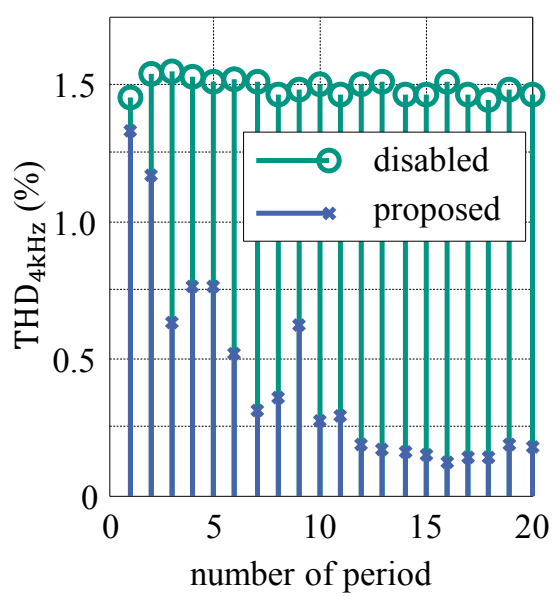

Fig. 4: Measurement of a torque step from minimal to maximal torque at half the nominal speed. Results of the proposed controller are given in blue and with disabled identification and memory blocks in green. Dynamic performance is comparable (a) but only the proposed controller holds the currents constant (b) yielding a very low $\mathrm{THD}_{4 \mathrm{kHz}}$ that is approached fast (c).

reference values significantly faster. After the reference currents are reached both controllers keep the currents constant. Therefore zoomed views are given in Fig. 4 (b) by means of the measured absolute currents.

$$
i_{\mathrm{abs}}=\sqrt{i_{\mathrm{d}}^{2}+i_{\mathrm{q}}^{2}}
$$

They are shown for 20 electric periods in dependence on the rotor angle $\gamma$ with the periods being counted as illustrated in Fig. 4 (a). As visible in the upper plot, the controller with disabled identification and memory blocks is not able to hold the currents constant due to the presence of voltage errors. In contrast, the proposed method successfully controls the currents to the constant reference values. Only measurement noise causes small current jittering. The proposed controller approaches the constant currents quickly. As can be seen in the lower plot of Fig. 4 (b) major deviations are only present in the first sixth of the first electric period. Thus, the precise identification of voltage errors and initialization of the memory yield fast convergence of the algorithm. This is further clarified

(a)

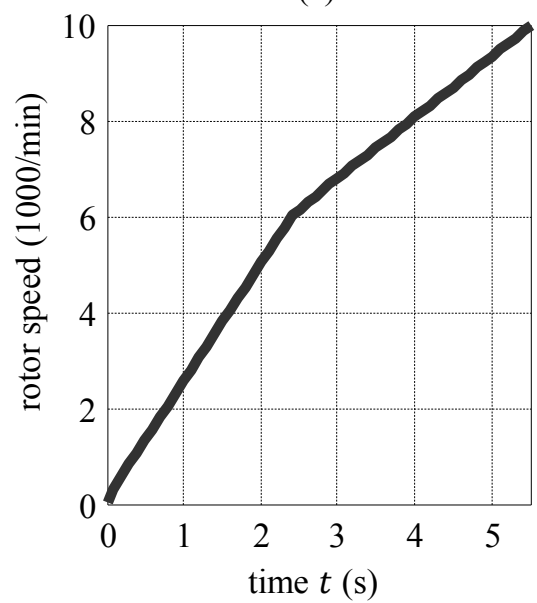

in Fig. 4 (c). The total harmonic distortion for frequencies up to $4 \mathrm{kHz}$ is calculated.

$$
\mathrm{THD}_{4 \mathrm{kHz}}=\frac{\sqrt{I^{2}-I_{1}^{2}}}{I_{1}}
$$

Therein $I$ denotes the effective phase current and $I_{1}$ the fundamental effective phase current. The $\mathrm{THD}_{4 \mathrm{kHz}}$ of the controller with disabled identification and memory blocks is about $1.5 \%$. The proposed controller reduces the $\mathrm{THD}_{4 \mathrm{kHz}}$ within several electric periods reaching its stationary value of about $0.2 \%$ after about $115 \mathrm{~ms}$.

\subsection{Variable Speed Operation}

As depicted in Fig. 5 (a) the machine under test is accelerated by the load machine from standstill to $10000 \mathrm{~min}^{-1}$ within $5.5 \mathrm{~s}$. During acceleration the reference currents of the machine under test are set constant to $i_{\mathrm{d}}^{*}=-150 \mathrm{~A}$ and $i_{\mathrm{q}}^{*}=0$ A so that current harmonics can be observed in variable speed operation. The measured absolute currents are shown for the controller

(b)

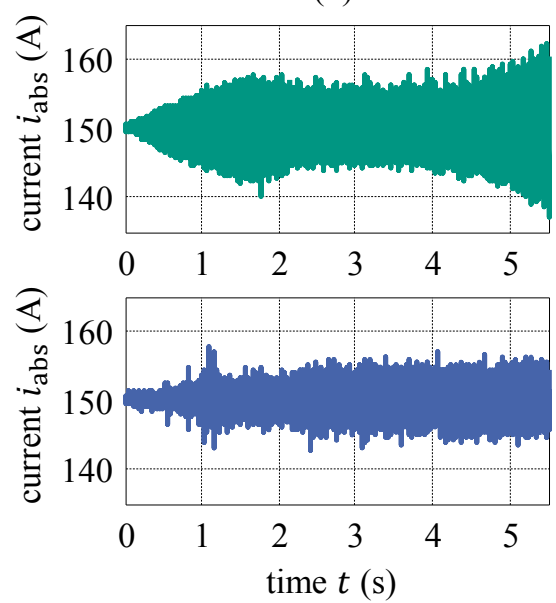

(c)

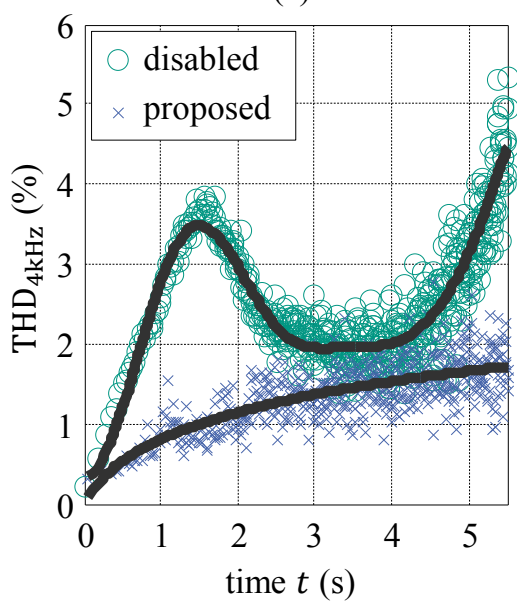

Fig. 5: Acceleration of the speed controlled load machine from standstill to $10000 \mathrm{~min}^{-1}$ (a) with constant reference currents $i_{\mathrm{d}}^{*}=-150 \mathrm{~A}$ and $i_{\mathrm{q}}^{*}=0 \mathrm{~A}$ of the machine under test. The proposed controller drawn in the lower plot of (b) mitigates current oscillations yielding a smaller total harmonic distortion $\mathrm{THD}_{4 \mathrm{kHz}}(\mathrm{c})$. 
with disabled identification and memory blocks in the upper plot and for the proposed controller in the lower plot of Fig. 5 (b). The proposed controller features lower current harmonics during acceleration due to its mitigation capability. However, the current ripple at $2100 \mathrm{~min}^{-1}$ is larger than the one of stationary operation in Fig. 4 (b). Although the controller is fast enough to mitigate harmonics even when the rotor speed changes, speed-introduced variations of the voltage errors hinder to the controller to reach the lower stationary current ripple. Otherwise it only takes about $100 \mathrm{~ms}$ to completely mitigate the current harmonics after the speed is constant. In order to compare the two controller setups in more detail the total harmonic distortion is calculated for each electric period and depicted in Fig. 5 (c). Because total harmonic distortion is calculated using a single electric period, the obtained data is noisy. That is why fits of the data are drawn as grey lines in the plot to illustrate the general trend of both curves. The proposed controller features a lower total harmonic distortion in the whole speed range. The value is reduced to about $28 \%$ at best at $1400 \mathrm{~min}^{-1}$ and $90 \%$ at worst at $3800 \mathrm{~min}^{-1}$.

\section{Conclusion}

The dynamics of a repetitive control based method to mitigate current harmonics in inverter-fed drives are analysed. The control method is found to mitigate all current harmonics that are integer multiples of the fundamental frequency, independent on their physical origin. Mitigation capability is only limited by the switching frequency and the voltage limit of the inverter. The method is based on online identification, rotor angle dependent storage and feed-forward control of voltage errors. Precise identification of voltage errors and improved initialization of the voltage error memory yields a fast dynamic response of the controller. Test bench experiments proof the validity of the underlying models. The total harmonic distortion at maximal torque and half the nominal speed can be reduced to about a seventh in comparison to the value of the standard mitigation method. The measured convergence of the algorithm is fast, reaching the stationary condition after several electric periods and within about $100 \mathrm{~ms}$ at typical operation.

Implementation of the method in existing inverters is easy. It can be realized by software updates because only the already available current and rotor angle sensors are needed. The method is microcontroller capable and extra need of calculation time and dynamic memory is little. The used functional principle is transferable to other machine types and grid applications enabling fast and precise mitigation of current harmonics in a wide field of applications.

\section{References}

[1] B. Francis, W. Wonham. "The internal model principle for linear multivariable regulators", Applied Mathematics and Optimization, 2, pp. 170-194 (1975).
[2] E. Kurniawan, Z. Cao, O. Mahendra, R. Wardoyo. "A survey on robust repetitive control and applications", Proc. IEEE Int. Conf. on Control System, Computing and Engineering, Batu Ferringhi, Malaysia, November 2014, pp. 524-529.

[3] M. Abusara, S. Sharkh, P. Zanchetta. "Adaptive repetitive control with feedforward scheme for grid-connected inverters", IET Power Electron., 8, pp. 1403-1410 (2015).

[4] D. Chen, J. Zhang, Z. Qian. "Research on fast transient and $6 n \pm 1$ harmonics suppressing repetitive control scheme for three-phase grid-connected inverters", IET Power Electron., 6, pp. 601-610 (2013).

[5] L. Ben-Brahim. "On the compensation of dead time and zero-current crossing for a PWM-inverter controlled AC servo drive", IEEE Trans. Ind. Electron., 51, pp. 11131118 (2004).

[6] J. Richter, T. Lannert, T. Gemassmer, M. Doppelbauer. "Mitigation of current harmonics in inverter-fed permanent magnet synchronous machines with nonlinear magnetics", Proc. PCIM Europe, Nuremberg, Germany, May 2015, pp. 725-732.

[7] P. Mattavelli, L. Tubiana, M. Zigliotto. "Torque-ripple reduction in PM synchronous motor drives using repetitive current control", IEEE Trans. Power Electron., 20, pp. 1423-1431 (2005).

[8] J. Richter, P. Bäuerle, T. Gemaßmer, M. Doppelbauer. "Transient trajectory control of permanent magnet synchronous machines with nonlinear magnetics", IEEE Int. Conf. on Industrial Technology, Seville, Spain, March 2015, pp. 2345 - 2351.

[9] J. Richter, M. Doppelbauer. "Mitigation and control of current harmonics in inverter-fed permanent magnet synchronous machines with nonlinear magnetics", IET Power Electron., submitted (2015).

[10] X. Chen, J. Wang, B. Sen, P. Lazari, T. Sun. "A highfidelity and computationally efficient model for interior permanent-magnet machines considering the magnetic saturation, special harmonics, and iron loss effect", IEEE Trans. Ind. Electron., 62, pp. 4044-4055 (2015).

[11] J. Richter, A. Dollinger, M. Doppelbauer. "Iron loss and parameter measurement of permanent magnet synchronous machines", Proc. Int. Conf. on Electrical Machines, Berlin, Germany, September 2014, pp. 16351641.

[12] N. Urasaki, T. Senjyu, T. Kinjo, T. Funabashi, H. Sekine. "Dead-time compensation strategy for permanent magnet synchronous motor drive taking zero-current clamp and parasitic capacitance effects into account", IEE Proc. Elect. Power Appl., 152, pp. 845-853 (2005).

[13] T. Sukagewa, K.Kamiyama, K. Mizuno, T. Matsui, T. Okuyama. "Fully digital, vector-controlled PWM VSIfed ac drives with an inverter dead-time compensation strategy", IEEE Trans. Ind. Appl., 27, pp. 552-559, (1991). 